\title{
INVESTIGATION OF ROBUST SHIP COURSE CONTROL BASED ON AUXILIARY LOOP METHOD
}

\author{
Aliya Imangazieva \\ Department of Higher and Applied Mathematics \\ Astrakhan State Technical University \\ Russian Federation \\ aliya111@yandex.ru
}

Article history:

Received 08.11.2021, Accepted 20.12.2021

\begin{abstract}
A novel robust control law is investigated on the problem of ship stabilization on the trajectory, which allows compensating perturbations in the parameters of the mathematical model of ship dynamics in cases of their changes caused by external conditions, namely: sea waves, currents, wind gusts, etc. To implement the proposed control law, only measured adjustable values are required such as the yaw angle and the control action that is the angle of the rudder of the ship. The synthesized ship course control system was investigated in the MATLAB. The law of controlling the ship's course with unknown parameters and external disturbances in the power supply is proposed. The design of the control law is based on a robust auxiliary loop algorithm and Khalil observers. The simulations illustrate the efficiency of the proposed control law.
\end{abstract}

\section{Key words}

Robust control, ship's course, auxiliary loop method, disturbance, Khalil observers.

\section{Introduction}

The ship's course control law experiences the disturbing effects of sea waves, currents and wind gusts. To effectively solve the problem of keeping the ship on a given trajectory, it is necessary to create a control law that would ensure the system's operability, compensating for the influence of disturbances, as well as parameter disturbances in cases of changes in the dynamic model of the ship caused by external conditions. Various approaches to the synthesis of the control law using various mathematical models are used to describe the dynamic processes and perturbations inherent in the ship [Gyoungwoo, Surendran and Sang-hyun, 2009]. Retention on a given trajectory using a PID controller based on neural networks is carried out in [Zeyu, Jiangqiang and Xingxing, 2012]. Such PID controllers in a number of developments provide the simplest parametric adjustment using a simplified model of angular motion [Lokukaluge, Guedes, 2013]. But the control laws based on a simplified model of angular motion do not take into account the wind drift of the ship, which can be significant. An adequate description of the real situation can be obtained using such models that take into account external disturbances of the water and air environments. Taking into account the moment of the impact of the wind, the angle of inclination of the wave, noise, as well as measurement errors, allow us to build a mathematical model of the ship, the parameters of which vary within certain limits [Dmitriev, Pelevin, 2004]. Thus, there is a need for a reliable control law. In addition, as noted in [Dmitriev, Pelevin, 2004], the PID controller generates a high-frequency nature of the control signal, which leads to increased wear of the steering drive.

Many methods of disturbance compensation for various control plants are proposed, for example, [Furtat, Orlov, 2020], [Furtat, 2013], [Morgun, Furtat, 2015]. Survey works on the history of development and the state of theoretical methods for constructing disturbance observers, as well as on their practical application, are [Andrievsky, Furtat, 2020a, 2020b].

One of the effective approaches that takes into account the uncertainty of the model parameters and the presence of disturbing factors caused by external conditions is robust control, proposed in [Tsykunov, Imangazieva, 2007]. The SISO system is developed by introducing an auxiliary loop and using observers of the system's derived signals. This article offers a solution to the problem of keeping a ship on a given trajectory under conditions of uncertainty, the action of external disturbances using this control algorithm. The mathematical model of the ship takes into account external disturbances: the moment of the impact of the wind, the angle of inclination 
of the wave, independent, generating white noise; measurement errors, as well as kinematic relations. The motion of the ship in the horizontal plane is considered, taking into account the lateral deviation from the trajectory. A reliable law for controlling the ship's course is constructed using the method of auxiliary loop [Tsykunov, 2007] and two Khalil observers [Atassi, Khalil, 1999].

\section{Robust control system with compensation of dis- turbances for a nonlinear plant}

Consider a plant model in the form

$Q(p) y(t)=k R(p) u(t)+\sum_{i=1}^{n} \sum_{j=1}^{q} p^{n-i} \varphi_{i j}(y(t)) \tau_{j}+f(t)$,

where $y(t), u(t)$ is a scalar adjustable variable and control, $p=d / d t$ is the differentiation operator, $f(t)$ is the disturbance, $Q(p), R(p)$ are normalized differential operators, $\operatorname{deg} Q(p)=n, \operatorname{deg} R(p)=m, k>0, \varphi_{i j}(y(t))$, $i=\overline{1, n}, j=\overline{1, q}$ are smooth functions satisfying the Lipschitz condition, $\tau_{j}$ are unknown constants.

The equation of the reference model is given

$$
Q_{m} y_{m}(t)=k_{m} r(t)
$$

where $r(t)$ is a setting influence, $k>0, y_{m}(t)$ is a scalar output, $\operatorname{deg} Q_{m}(t)=n-m$.

The proposed control law should ensure fulfillment of the goal

$$
\left|y(t)-y_{m}(t)\right|<\delta
$$

where $\delta>0$ is a required accuracy.

\section{Assumptions.}

1. Coefficients of the polynomials $Q(p), R(p)$, parameters $k, \tau_{j}$ belong to a known compact set $\Xi$.

2. $\varphi_{i j}(y(t)), i=\overline{1, n}, j=\overline{1, q}$ are smooth functions satisfying the Lipschitz condition.

3. $f(t), r(t)$ is bounded functions.

4. $R(\lambda), Q_{m}(\lambda)$ are Hurwitz polinomials, where $\lambda$ is complex variable.

5. The orders of polinomials $Q(p), R(p)$ are known, $\operatorname{deg} Q(p)=n, \operatorname{deg} R(p)=m$.

Applying the control law proposed in the paper [Imangazieva, Tsykunov, 2007] we have the following closed system

$$
\left\{\begin{array}{l}
\text { Control plant: } \\
Q(p) y(t)=k R(p) u(t)+\sum_{i=1}^{n} \sum_{j=1}^{q} p^{n-i} \varphi_{i j}(y(t)) \tau_{j}+f(t) . \\
\text { Control low: } u(t)=T \varsigma, T=\left[l_{0}, l_{1}, \ldots, l_{n-m-1}\right], \\
T(\lambda) / Q_{m}(\lambda)=1 /(1+\lambda) . \\
\text { Observer 1: } \dot{\varsigma}=F_{0} \varsigma(t)+B_{0}(v(t)-\bar{v}(t)), \\
\bar{v}(t))=L \varsigma(t), \varsigma \in R^{n-m}, F_{0}-\text { frobenius matrix, } \\
L=[1,0, \ldots 0], B_{0}^{T}=\left[b_{1} / \mu, \ldots, b_{n-m} / \mu^{n-m}\right], \\
F=F_{0}+B L-\text { hurwitz matrix, } B^{T}=\left[b_{1}, \ldots, b_{n-m}\right] . \\
\text { Auxiliary loop: }\left(p+a_{m}\right) \bar{e}(t)=\beta v(t), \zeta(t)=e(t)-\bar{e}(t) . \\
\text { Observer } 2: \dot{z}(t)=\bar{F}_{0} z(t)+\bar{B}_{0}(\zeta(t)-\bar{\zeta}(t)), \\
\bar{\zeta}(t)=L_{2} z(t), z \in R^{2}, \bar{F}_{0}-\text { frobenius matrix, } \\
L_{2}=[1,0], \bar{B}_{0}^{T}=\left[b_{1} / \mu, b_{2} / \mu^{2}\right], v(t)=-\frac{1}{\beta} c z, c=\left[a_{m}, 1\right] .
\end{array}\right.
$$

\section{Plant model and Problem statement}

Papers [Dmitriev, Pelevin, 2004] provide a complete system of equations used in solving the problem of stabilizing a ship on a trajectory and including equations of ship dynamics, external disturbances and errors of change, as well as kinematic relations. The movement of the ship in the horizontal plane is considered, taking into account the side swing. Mathematical model of plant represents its description in space of states in the form of nonlinear matrix differential equation [Dmitriev, Pelevin, 2004]

$$
\dot{X}=F X+P(X) X+C U+G W,
$$

where $F$ is a matrix of dynamic, $P(X) X$ is a vector nonlinear members, $C$ is a matrix of control, $W$ is the unit intensity vector white noise process, $G$ is a matrix of perturbations.

State vector $X=\left(V_{y}, \omega_{z}, \eta(t), \psi, f, m\right)$ :

$V_{y}$ is a lateral relative velocity;

$\omega_{z}$ is a yaw angular velocity;

$\eta$ is a lateral deviation from trajectory;

$\psi$ is a yaw angle;

$f$ is a reduced force;

$m$ is a moment of wind impact.

Perturbation vector: $W=\left(w_{1}, w_{2}, \alpha_{p}, \dot{\alpha}_{p}\right)$, where $w_{1}, w_{2}$ are independent components, generating white noise of unit intensity, yaw angle of wave slope, model of which will be described in section 3.1.

Control: $u=\delta$ is the steering angle.

Output of the plant: $y=K$ is the ship's course angle.

Output of reference model: $y_{m}=P$ is the set course angle.

Controlled variable: $e(t)=\psi(t)=y-y_{m}$ is the yaw angle.

Also in paper matrix, including in equation (3), for a three-point lateral wave:

$$
\begin{aligned}
F & =\left(\begin{array}{cccccc}
-0,045 & -3,44 & 0 & 0 & 0 & 0 \\
0,006 & -0,346 & 0 & 0 & 0 & 0 \\
1 & 0 & 0 & 4,8 & 0 & 0 \\
0 & 1 & 0 & 0 & 0 & 0 \\
0 & 0 & 0 & 0 & -0,0003 & 0 \\
0 & 0 & 0 & 0 & 0 & -0,0003
\end{array}\right), \\
G & =\left(\begin{array}{cccc}
0,0012 & 0 & 0 & 0 \\
0 & 0,0001 & 0 & 0 \\
0 & 0 & 0 & 0 \\
0 & 0 & 0 & 0 \\
0 & 0 & 0,000061 & 0 \\
0 & 0 & 0 & 0,00004
\end{array}\right)
\end{aligned}
$$




$$
\begin{aligned}
& P(X)=\left(\begin{array}{cccccc}
0,02\left|V_{y}\right| & 0 & 0 & 0 & 0 & 0 \\
0 & 0 & 0 & 0 & 0 & 0 \\
0 & 0 & 0 & 0 & 0 & 0 \\
0 & 0 & 0 & 0 & 0 & 0 \\
0 & 0 & 0 & 0 & 0 & 0 \\
0 & 0 & 0 & 0 & 0 & 0
\end{array}\right), \\
& C=\left(\begin{array}{c}
-0,175 \\
0,25 \\
y 0 \\
0 \\
0 \\
0
\end{array}\right) \text {. }
\end{aligned}
$$

Then equation (4) in the operator form gives a description of the plant by the form input-output:

$$
\begin{gathered}
p^{6}+0,3916 p^{5}-0,00483531 p^{4}-0,300681 \cdot 10^{-5} p^{3}- \\
\left.-0,4563 \cdot 10^{-9} p^{2}\right) y(t)=\left(0,025 p^{4}+0,00219 p^{3}+\right. \\
\left.\quad+0,130725 \cdot 10^{-5} p^{2}+0,19575 \cdot 10^{-9} p\right) u(t)+ \\
+\left(0,012 p^{5}+0,0004 p^{4}\right)\left(x_{1}-y_{1}\right)+\left(-0,344 \cdot 10^{-3} \cdot p^{4}-\right. \\
\left.\quad-0,2 \cdot 10^{-6} p^{3}\right)\left(x_{2}-y_{2}\right)+0,61 \cdot 10^{-4} .^{4}+ \\
\left.\quad+0,2112 \cdot 10^{-4} p^{3}+0,1 \cdot 10^{-7} p^{2}\right) w_{1}(t)+ \\
\left.\left.\quad+\left(-0,1376 \cdot 10^{-3} p^{3}\right) w_{2}(t)+0,02\right\rfloor V_{y}\right\rfloor p^{5},
\end{gathered}
$$

where according Assumptions

$Q(p)=p^{6}+0,3916 p^{5}-0,00483531 p^{4}-0,300681$. $\left.10^{-5} p^{3}-0,4563 \cdot 10^{-9} p^{2}\right)$,

$R(p)=0,025 p^{4}+0,00219 p^{3}+0,130725 \cdot 10^{-5} p^{2}+$ $0,19575 \cdot 10^{-9} p$.

Target condition (3) has the form

$$
|\psi(t)|<\delta
$$

starting from a certain moment of time $T$, where $\delta$ is required accuracy.

For the ship plant, the yaw angle $\psi$ in the horizontal plane is stabilized, taking into account the lateral deviation $\eta$.

\subsection{Perturbations model}

The force and moment of the wave action are expressed in terms of the angle of the wave slope $\alpha_{p}$. Adequate model of the real situation can be obtained by describing the angle of the wave slope as follows [Dmitriev, Pelevin, 2004]

$$
\alpha_{p}=x_{1}-y_{1}, \dot{\alpha}_{p}=x_{2}-y_{2},
$$

where $x_{i}, y_{i}, i=1,2$ described by a system of differential equations

$$
\begin{aligned}
& \dot{x}_{1}=x_{2}, \\
& \dot{x}_{2}=-a_{1} x_{2}-a_{2} x_{1}+c_{x} w_{4}, \\
& \dot{y}_{1}=y_{2}, \\
& \dot{y}_{2}=-b_{1} y_{2}-b_{2} y_{1}+b_{2} x_{1}+b_{1} x_{2},
\end{aligned}
$$

course control system the GK gyrocompass is rigidly connected to the controlled plant ship and through it the main negative feedback is carried out on the deviation of the ship from the given course [Vlasenko, Guardian,1983]. The true course, measured with the GK and the SD selsyn-sensor, is transmitted through the SR selsyn-receiver and the IT irreversible transmission to the MD mechanical differential, which is an element of comparison. The set value of the course is fed to the second shaft of the MD. On the output (third) shaft of the $\mathrm{MD}$, the difference between the set and true values of the course is obtained, i.e. the angle of deviation of the ship from the given course $e(t)=y(t)-y_{m}(t)$. The output shaft of the MD turns the converting device - the selsyn 
course sensor SCS, operating in transformer mode. It converts the angle of deviation of the ship from a given course into an electrical signal, which is fed to the input of the amplifier of the SG steering gear.

Further improvement of the steering wheels is on the way to replacing individual elements of the scheme with more modern ones. The control algorithm proposed in the functional scheme of the autoroule as a block for forming a control algorithm BFAU.

Following the control law described in section 2, we will set the law of change $u(t)$ in the form

$$
u(t)=T \varsigma(t)
$$

where $\varsigma(t)$ - state vector of Observer 1, described by the following equations

$$
\begin{aligned}
& \dot{\varsigma}_{1}(t)=\varsigma_{2}(t)+600\left(\bar{v}(t)-\varsigma_{1}(t)\right), \\
& \dot{\zeta}_{2}(t)=90000\left(\bar{v}(t)-\varsigma_{1}(t)\right), \bar{v}(t)=\varsigma_{1}(t)
\end{aligned}
$$

After applying parameterization, selecting an operator $T(p)$ that satisfies the condition $T(p) / Q_{m}=1 /(p+$ 0,01 ) equation (4) is transformed to the form

$$
(p+0,01) e(t)=v(t)+\phi(t) .
$$

The signal $\phi(t)$ contains all the uncertainty of the parameters of the control plant, nonlinearity and external disturbances, the model of which is described in section 3.1.

Introduce an auxiliary loop to compensate the signal $\phi(t)$

$$
(p+0,01) \bar{e}(t)=v(t) .
$$

and we will make an equation for the mismatch $\zeta(t)=$ $e(t)-\bar{e}(t)$, taking into account (10),(11)

$$
(p+0,01) \zeta(t)=\phi(t) .
$$

The signal $v(t)$ is formed in the form

$$
v(t)=-\left(\frac{1,0001 p+0,01}{0,01 p+1}\right) \bar{\zeta}(t)
$$

where $\bar{\zeta}(t)$ estimate obtained from Observer 2, presented in the BFAU as a real-differentiating link of the form $\frac{p}{0,01 p+1}$.

\section{Results of a numerical simulation of the ship's course control system}

In accordance with accepted maritime practice, the line of a given path is represented as a set of rectilinear sections. To solve the problem of controlling the ship's course, the trajectory consisting of two rectilinear sections. Numerical simulation of the control system was carried in the MatLab Simulink software environment. Figure 1 shows the trajectory of the ship corresponding to the reference model. The driving time is 600 seconds at a speed of $5 \mathrm{~m} / \mathrm{s}$. The motion graph is presented in the trajectory coordinate system $O \xi \eta$.

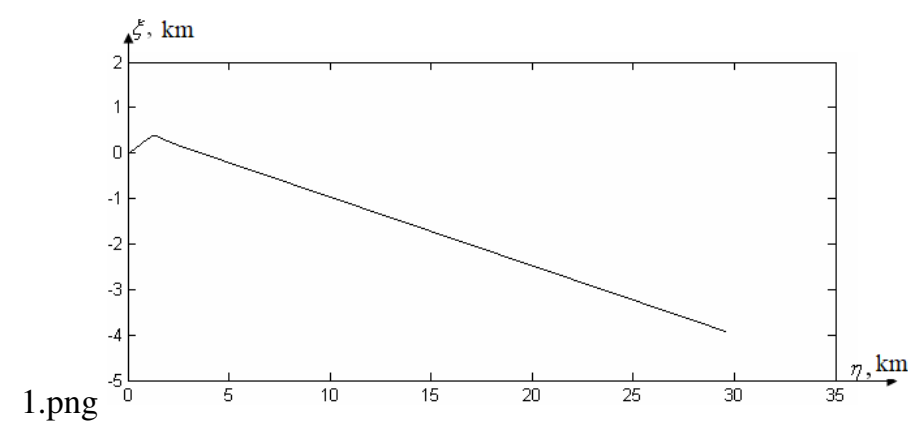

Figure 2. The trajectory of the ship

For a three-point lateral wave, the following transient processes are obtained for the tracking error, control and lateral deviation. Figures 3,4,5 show the transition process of the tracking error (yaw angle ) $e(t)=\psi(t)=$ $y(t)-y_{m}(t)$, [rad], transition process of the lateral deviation $\eta(t)$, [m], transition process of the control action (steering angle) $u(t)=\delta(t)$,[rad].

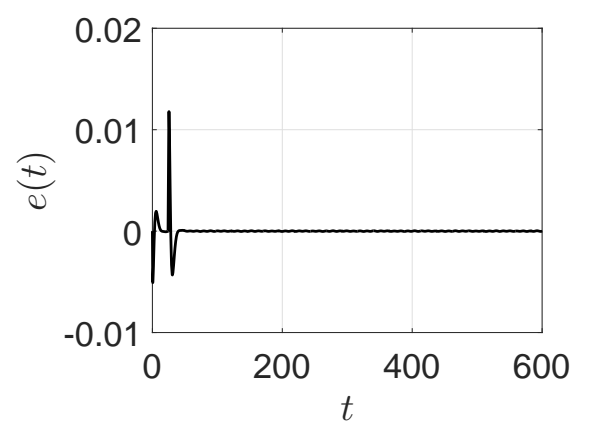

Figure 3. The transition process of the tracking error $e(t)=\psi(t)=y(t)-y_{m}(t),[\mathrm{rad}]$

\section{Conclusion}

The problem of keeping a ship on a given trajectory under conditions of uncertainty, the action of external disturbances is investigated. A robust control low is applied for a plant-a ship, the model of which takes into account the moment of wind impact, the angle of the wave slope, the independent ones that generate white noise; measurement errors, as well as kinematic relations. The results of numerical simulation in Matlab Simulink are presented. The efficiency of the algorithm on a trajectory consisting of two rectilinear sections is shown. 


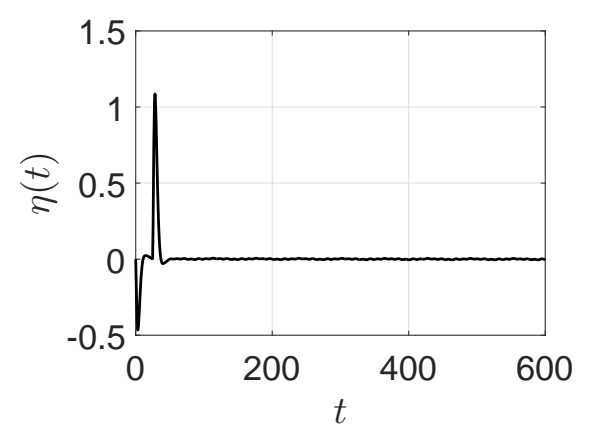

Figure 4. The transition process of the lateral deviation $\eta(t),[\mathrm{m}]$

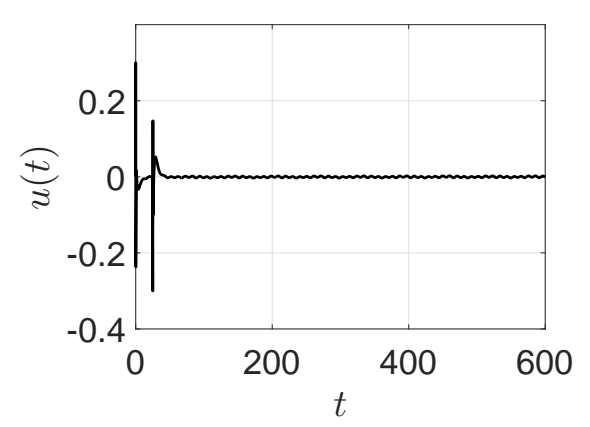

Figure 5. The transition process of the control action (steering angle) $u(t)=\delta(t),[\mathrm{rad}]$

\section{Acknowledgment}

The reported study was funded by RFBR, project number 20-08-00610.

\section{References}

Andrievsky, B.R., Furtat, I.B. (2020) Disturbance Observers: Methods and Applications. I. Methods. Autom. Remote Control 81 (9), pp. 1563-1610.

Andrievsky, B.R., Furtat, I.B. (2020) Disturbance Observers: Methods and Applications. II. Applications. Autom. Remote Control 81 (10), pp. 1775-1818.

Atassi, A.N., Khalil, H.K.(1999) Separation principle for the stabilization of class of nonlinear systems. IEEE Trans. Automat. Control, 44 (9), pp. 1672-1687.

Borodai, I.K., Netsvetaev, U.A.(1982) Seaworthiness of vessels. Sudostroenie, Leningrad. (in Russian).

Dmitriev, S.P., Pelevin, A.E. (2004) Problems of navigation and control during the stabilization of the vessel on the trajectory. CSII. Electropribor,Spb. (in Russian).

Furtat, I.B. (2020) Synchronization and state estimation of nonlinear systems with unknown time-delays: adaptive identification method. Cybernetics and Physics, 9(3), pp. 136-143.

Furtat, I. (2016) Robust synchronization of the structural uncertainty nonlinear network with delay and disturbances1. IFAC Proceedings Volumes (IFACPapersOnline), 11(PART), pp. 227-232.

Gyoungwoo, Lee., Surendran, S., Sang-Hyun, Kim. (2009) Algorithms to control the moving ship during harbour entry. Applied Mathematical Modelling. 33(5), pp. 2474-2490.

Lokukaluge, L.P., Guedes, S. (2013) Lyapunov and Hurwitz based controls for input-output linearisation applied to nonlinear vessel steering. Ocean Engineering, 66, pp. 55-68.

Margun, A., Furtat, I. (2015) Robust control of linear MIMO systems in conditions of parametric uncertainties, external disturbances and signal quantization. 20th International Conference on Methods and Models in Automation and Robotics, MMAR 5(2015), pp. 341-346.

Tsykunov, A.M., Imangazieva, A.V. (2007) The robust control of linear dynamyc plant with time delay on state. Mekhatronika, Avtomatizatsiya, Upravlenie 12, pp. 2-6. (In Russian)

Tsykunov, A.M. (2007) Robust Control Algorithms with Compensation for Bounded Perturbations. Autom. Remote Control 68 (7), pp. 1213-1224.

Vlasenko, A.A., Guardian, V.A. (1983) Marine electrical automation. Transport, Moscow. (in Russian).

Zeyu, L., Jiangqiang, H., Xingxing, H. (2012) PID control based on RBF neural network for ship steering. WICT Trivandrum, India, 30 oct.-2 nov.pp. 10761080. 